\title{
A Pilot Trial of Vascular Targeted Photodynamic Therapy for Renal Tissue
}

\author{
Surena F. Matin, ${ }^{*} \dagger$ Peggy T. Tinkey, Agatha T. Borne, L. Clifton Stephens, Avigdor Sherz \\ and David A. Swanson \\ From the Departments of Urology (SFM, DAS), Veterinary Medicine and Surgery (PTT, ATB, LCS), University of Texas M. D. Anderson \\ Cancer Center, Houston, Texas, and Department of Plant Science, Weizmann Institute of Science (AS), Rehovot, Israel
}

Purpose: Vascular targeted photodynamic therapy represents the newest generation of photodynamic therapy and a new paradigm for minimally invasive ablative therapy. We report a pilot trial of vascular targeted photodynamic therapy to evaluate the effect on porcine renal tissue.

Materials and Methods: Pigs underwent continuous infusion of WST-09 (Negma-Lerads, Toussous le Noble, France) and concurrent illumination with interstitial laser at a wavelength of $763 \mathrm{~nm}$ to the lower pole of the kidney. Drug doses were 0.5 to $1.0 \mathrm{mg} / \mathrm{kg}$ and light doses were 100 to $200 \mathrm{~J}$. Nuclear renography was performed on postoperative day 5 . On postoperative day 7 arteriography, pyelography, computerized tomography of the abdomen and necropsy were performed.

Results: Four of 7 animals completed therapy and all evaluations. Three animals died, including 1 of surgical complications and 2 of an anaphylactoid reaction to the Cremophor® solvent in the compound. All kidneys in surviving animals functioned on nuclear renography. Renal function remained unchanged. No lesions or urine leakage was visible on imaging. On necropsy lesion size was $5 \times 4 \times 3$ to $7 \times 7 \times 14 \mathrm{~mm}$ depending on the drug/light dose. Histology showed a distinct demarcation between the treated zone and the surrounding parenchyma at higher doses. Lesions were well demarcated with necrotic tubules, glomerular fibrinoid necrosis, capillary loop thrombosis, interstitial hemorrhage and lymphocytic infiltrates.

Conclusions: Significant tissue effect with some necrosis was seen at these low drug/light combinations. This study provides the initial proof of principle that justifies further preclinical investigation of vascular targeted photodynamic therapy for renal tumors. A newer, water based formulation should decrease the incidence of reactions in swine. This newer formulation may allow further safe investigation of this novel treatment paradigm.

Key Words: kidney; carcinoma, renal cell; photochemotherapy; lasers; swine

$\mathrm{P}$ hotodynamic therapy is a unique form of therapy by which a photochemical reaction generates free radicals with the capability of causing tissue injury and necrosis. PDT is much different from other modalities using laser energy, such as interstitial laser coagulation, laser thermal ablation or laser interstitial thermal therapy, which generate thermal energy. In the latter cases heat is the primary cause of tissue ablation, whereas with PDT the generation of ROS causes tissue injury. Currently approved indications for PDT include age related macular degeneration, skin cancer, other cutaneous disorders, brain, lung, esophageal, gastric and bladder cancer, cervical dysplasia and cervical cancer. ${ }^{1}$ New areas of investigation include its use for endovascular applications. ${ }^{2}$

Clinical experience with PDT in urology is limited. Until recently the major role of PDT in urology has been for the

Submitted for publication October 23, 2007.

Study received institutional animal safety committee approval.

Supported by Cancer Center (CORE) Support Grant NIH-NCI CA-16672 and Negma-Lerads/Steba Biotech.

* Correspondence: Department of Urology, 1515 Holcombe Blvd., Unit 1373, Houston, Texas 77030 (telephone: 713-792-3250; FAX: 713-794-4824; e-mail: surmatin@mdanderson.org).

$\dagger$ Financial interest and/or other relationship with Ethicon BioSurgicals.

$¥$ Financial interest and/or other relationship with Kidney Cancer Association, Bayer/Onyx and Pfizer. diagnosis and treatment of bladder cancer but it is currently undergoing phase II/III clinical trials in patients with primary prostate cancer in whom radiation therapy failed. ${ }^{3-5}$ The shortcomings of current popular photosensitizers have significantly held back development, including the use of shorter wavelengths (which have less tissue penetration), phototoxicity requiring prolonged avoidance of sun exposure and slow clearance.

A new generation of PDT is now available with unique properties that are advantageous in comparison to those of traditional PDT. These properties include rapid clearance, resulting in minimal phototoxicity and sequestration in the vascular compartment, which may have some advantages from a selectivity and toxicity perspective. ${ }^{6-8}$ Unique targeting by the new generation of PDT agents has motivated this application to be termed VTP. Based on this recent availability of VTP we performed a pilot trial evaluating its safety and efficacy for treating renal tissue.

\section{MATERIALS AND METHODS}

\section{Animal Care and Surgical Procedures}

This study was approved by the institutional animal safety committee. Animals were maintained in facilities approved by the Association for Assessment and Accreditation of Laboratory Animal Care, International and in accordance with 
current United States Department of Agriculture, Department of Health and Human Services, and National Institutes of Health regulations and standards. Domestic female juvenile pigs weighing $30 \mathrm{~kg}$ were obtained from a local breeder and held a minimum of 7 days for acclimation and observation before enrollment on the protocol. Any pigs with clinical signs of disease, such as anorexia, diarrhea, nasal discharge, coughing, etc, were rejected and removed from the facility. Food was withheld for at least 12 hours before surgery, while water was available at all times.

Pigs were premedicated intramuscularly with atropine $(0.04 \mathrm{mg} / \mathrm{kg})$ and sedated intramuscularly with ketamine (30 $\mathrm{mg} / \mathrm{kg})$ plus acepromazine $(0.3 \mathrm{mg} / \mathrm{kg})$. They were then intubated and maintained on $1 \%$ to $3 \%$ isoflurane in oxygen. The pigs were mechanically ventilated at 10 to 12 breaths per minute with a tidal volume of 10 to $15 \mathrm{ml} / \mathrm{kg}$. An intravenous catheter was placed in the auricular vein and the external jugular vein. Intravenous fluids $(0.9 \% \mathrm{NaCl})$ were given throughout surgery. Pigs were under stable anesthesia for an average of 30 minutes before beginning the procedure.

After inducing general anesthesia the animal was placed in the left lateral decubitus position and laparoscopic access was obtained with a Veress needle. Pneumoperitoneum was established at a pressure of $15 \mathrm{~mm} \mathrm{Hg}$. One $12 \mathrm{~mm}$ and 2, 5 $\mathrm{mm}$ ports were placed. The lower pole of the right kidney was exposed and a laser fiber was inserted $1 \mathrm{~cm}$ into the anterior lower pole. Treatment was initiated 5 minutes after WST-09 injection was started. After treatment was completed the laser fiber was withdrawn, all laparoscopic ports were removed and the skin was closed using wire staples. Animals received standard postoperative care, including analgesia, antibiotics and routine postoperative evaluations.

\section{Photosensitizer Preparation, Laser Methodology and PDT Delivery}

WST-09 (Tookad® or palladium-bacteriopheophorbide) was supplied as a dark violet liquid in sterile glass vials. The compound was stored at $4 \mathrm{C}$ in fully darkened conditions, and all vials and delivery devices (intravenous tubing, connectors, syringes, etc) were wrapped in aluminum foil to prevent exposure to ambient light. All drug manipulation occurred at low light conditions, including darkening of the operating room and lowering the laparoscopic light source to the lowest possible intensity. Intravenous injection of WST-09 was performed by continuous infusion using a digital micro-injector programmed to deliver the entire dose steadily during 20 minutes. The drug doses used were 0.5 and $1.0 \mathrm{mg} / \mathrm{kg}$.

Five minutes after the initiation of drug infusion a $600 \mu$ silica laser fiber fitted with a diffuser tip producing a homogeneous radial light pattern was introduced transcutaneously and inserted $1 \mathrm{~cm}$ into the lower pole of the kidney. Interstitial illumination was initiated at a wavelength of 763 nm using a Ceralas® PDT 763 medical laser system. The light doses used were 100 and $200 \mathrm{~J}$. Light fluence was kept low as to avoid any confounding effects from thermal injury. ${ }^{9}$ However, it is possible that, when light excitation is performed on circulating sensitizers, such as with WST-09, the thermal effect is minimized, allowing higher light regimens, but we have not had the opportunity to evaluate this. ${ }^{10}$ Figure 1 shows the treatment design.

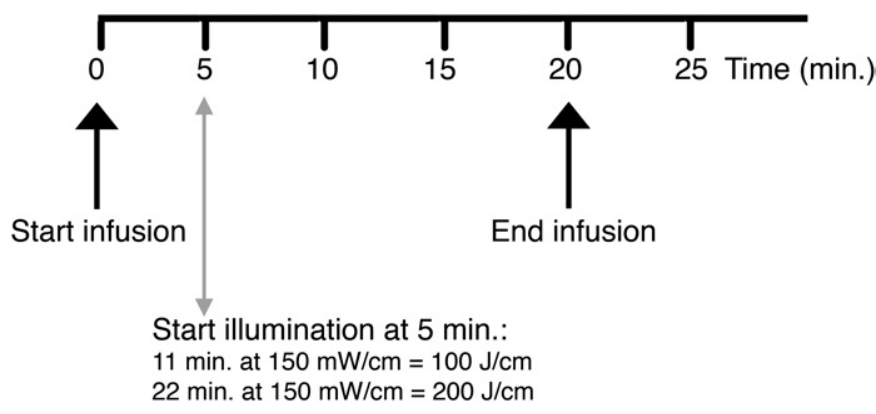

FIG. 1. Timeline of drug infusion and illumination parameters

\section{Evaluation During and After Treatment}

Laboratory studies, consisting of a complete blood count, serum electrolytes, blood urea nitrogen and creatinine tests, were performed on the day of surgery just before treatment, and on PODs 5 and 7. Nuclear renography was performed on POD 5 using ethylene dicysteine ${ }^{99}$ technetium to evaluate differential renal function. On POD 7 arteriography, pyelography and renal protocol CT of the abdomen were performed just before necropsy. The animals were sacrificed by exsanguination under deep anesthesia at the completion of the final imaging study.

Necropsy and histology evaluation was performed by a single veterinary pathologist (LCS) who was blinded to treatment parameters and uninvolved with treatment. After exploratory laparotomy the treated kidney was harvested. The treatment zone was readily identified visually. The kidney was bisected in 2 dimensions perpendicular to the surface where the treatment occurred, and the lesion was measured in 3 dimensions. Photographs were taken and tissue samples were harvested in formalin for histological evaluation. Histology was performed by standard hematoxylin and eosin staining.

\section{RESULTS}

Seven animals underwent the experiments. Two of the first 5 animals died within minutes of starting the drug infusion. Another 2 animals required medical management for hypotension during drug infusion. Before proceeding further all possibilities of these events were evaluated and it was believed that the animals had experienced an anaphylactoid reaction to the Cremophor solvent in the compound. Subsequent animals were treated proactively with generous intravenous hydration and medical management. These steps prevented any further deaths. One animal with bowel injury during initial laparoscopic access was sacrificed before drug administration. Overall 4 animals completed treatment and all evaluations before sacrifice. All kidneys in the 4 animals completing therapy functioned on nuclear renography on POD 5 (fig. 2, A). Laboratory studies remained stable and within normal limits. Imaging before sacrifice on POD 7 showed no visible lesions on arteriography or CT and no urine leakage was seen on retrograde pyelography in any surviving animals (see table).

On necropsy the treatment zone was clearly visible as a depression of the capsular surface without any surrounding organ damage (fig. $2, B$ ). Histological evaluation of the lesion at the cortical level showed widespread loss 

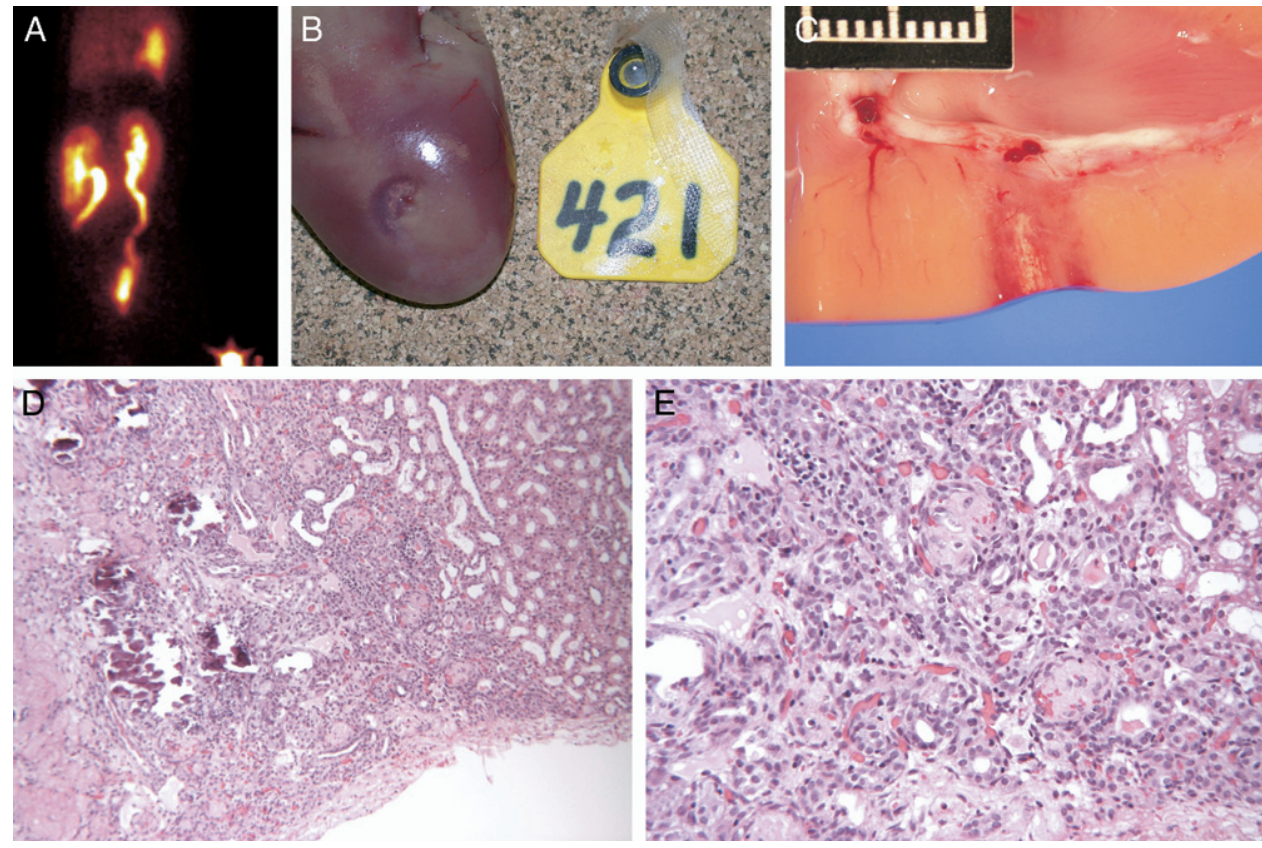

Fig. 2. Animal 421. A, nuclear renogram on POD 5 shows symmetrical renal function. $B$, photograph of kidney 1 week after receiving 0.5 $\mathrm{mg} / \mathrm{kg}$ WST-09 and $200 \mathrm{~J}$ laser light dose. Cortical depression indicates treatment site. $C$, bisection of kidney through treatment zone reveals pale central column of necrosis surrounded by hyperemic zone. $D$, photomicrograph of section at capsule depression site demonstrates lesion margin with viable kidney (right), zone of basophilia composed of inflammation and mineralization, and necrotic renal parenchyma (left corner). Reduced from $\times 100$. $E$, this region of lesion shows tubular loss, glomerular necrosis and diffuse lymphocytic inflammation. Reduced from $\times 250$.

and necrosis of tubules (fig. $2, D$ and $E$ ). Outright coagulative necrosis was seen in the central portion of the outer cortex, surrounded by an accumulation of granular basophilic material that was compatible with mineral deposition. The most significant and dramatic changes occurred to glomeruli, which were lost (outright necrosis) or had significant hemorrhage and thrombosis of the capillary loops. However, there was no complete necrosis of all central tissue. At the edge of the treatment zone the glomerular lesions were segmental, affecting only portions of the glomerular tuft. In the cortex and medulla the remaining tubules were filled with blood or hyaline casts. Some were seen to show enlargement and epithelial basophilia along with poor polarity, indicating attempts at repair. The interstitium of the lesion was infiltrated diffusely by lymphocytes with the most intense lymphocytic infiltrates seen in the medulla. Arterioles in the interstitium showed hypertrophy of the smooth muscle of the tunica media as well as endothelial cell enlargement.

\section{DISCUSSION}

This study demonstrates certain findings. 1) Cremophor, which was required as a solvent for the photosensitizer, may cause an anaphylactoid reaction in swine. After this was discovered it was readily managed by appropriate intraoperative maneuvers. 2) VTP can otherwise be delivered safely in targeted fashion to a specific location in the kidney without surrounding damage to nearby structures and without discernible effects on renal function. 3) Tissue effects were seen with every treatment and they seemed to follow a dose response (fig. 3), although this conclusion is based on limited data. 4) These relatively low drug/light dose combinations were insufficient to create reproducible homogeneous necrosis in normal kidney tissue. The use of higher light/drug doses and multiple fibers should amplify necrosis and the effect on malignant tissue may potentially be more dramatic, although we are not yet able to test these hypotheses.

PDT relies on the 3 interacting components of light, drug and molecular oxygen. The drug is a photosensitizer that is usually delivered intravenously. Light of a specific wavelength generates ROS from the photosensitizer. ROS cause organelle (mitochondria), cellular and microvascular damage via the oxidation of lipids, lipoproteins and proteins. ${ }^{11}$ Membrane injury induces apoptosis if the mitochondrial membrane is injured by protease release,

\begin{tabular}{|c|c|c|c|c|c|c|c|c|}
\hline \multirow[b]{2}{*}{ Animal No. } & \multicolumn{2}{|c|}{ Dose } & \multirow[b]{2}{*}{ Status } & \multicolumn{3}{|c|}{ Day 7 Results } & \multirow{2}{*}{$\begin{array}{l}\text { Day 0/5/7 Serum } \\
\text { Creatinine (mg/dl) }\end{array}$} & \multirow{2}{*}{$\begin{array}{l}\text { Day } 7 \text { Lesion Length } \times \\
\text { Width } \times \text { Depth }(\mathrm{mm})\end{array}$} \\
\hline & Drug (mg/kg) & Light $(\mathrm{J})$ & & $\mathrm{CT}$ & Arteriography & Pyelography & & \\
\hline 379 & 0.5 & 100 & Died of surgical complication & - & - & - & - & - \\
\hline 421 & 0.5 & 200 & Survived & Neg & $\mathrm{Neg}$ & $\mathrm{Neg}$ & $1.4 / 1.4 / 1.2$ & $7 \times 7 \times 7$ \\
\hline 380 & 1.0 & 100 & Died of hypotension & - & - & - & - & - \\
\hline 422 & 1.0 & 100 & Survived & Neg & Neg & $\mathrm{Neg}$ & $1.3 / 1.2 / 1.3$ & $7 \times 10 \times 7$ \\
\hline 423 & 1.0 & 200 & Survived & Neg & Neg & $\mathrm{Neg}$ & $1.4 / 1.3 / 1.6$ & $7 \times 7 \times 14$ \\
\hline
\end{tabular}




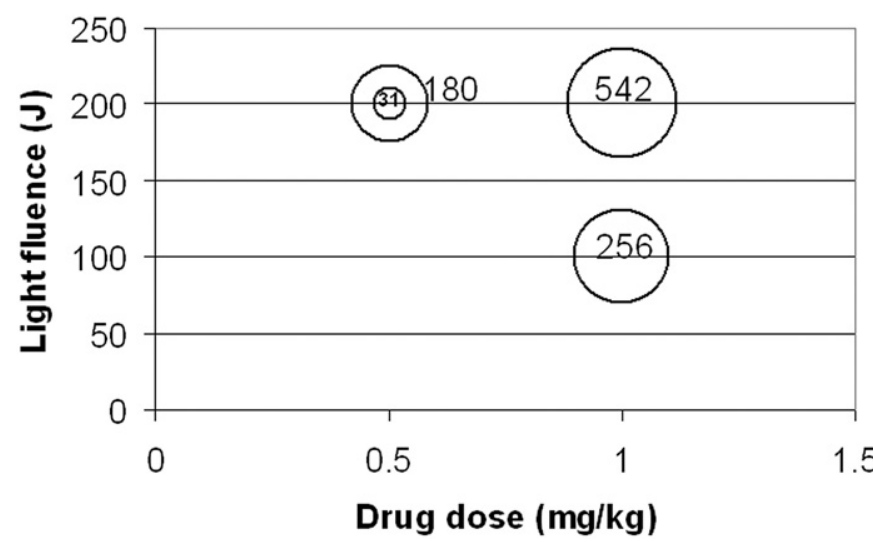

FIG. 3. Bubble graph demonstrates relationship of drug dose, light fluence and therapy lesion volume. Relative size of circles indicates volume. Numbers in circles indicate $\mathrm{mm}^{3}$. Volume was calculated using formula, $4 / 3 \pi \times$ radius $1 \times$ radius $2 \times$ radius $3 .{ }^{21}$

whereas if the cell membrane is injured, necrosis ensues due to cell lysis. ${ }^{12}$ Vascular damage and collapse are also an important mechanism of tissue destruction, in addition to cytotoxicity and apoptosis. ${ }^{12,13}$ These initial experiments were designed to allow these physiological processes to occur during a week before tissue evaluation.

The history of PDT started with initial studies at Mayo Clinic almost 50 years ago using hematoporphyrin derivatives. ${ }^{14}$ Subsequent clinical development and experience with porphyrin based PDT was performed by researchers at Roswell Park Cancer Institute, leading to the development of the first-generation photosensitizer. ${ }^{15}$ This compound, porfimer sodium (Axcan Scandipharm, Birmingham, Alabama), absorbs light at less than $640 \mathrm{~nm} .{ }^{15}$ At this wavelength light does not penetrate tissues deeply, partly because of tissue pigmentation and absorption by endogenous porphyrins..$^{9}$ In addition to poor penetration, other limitations of the first-generation photosensitizers are a long half-life and significant, prolonged skin phototoxicity.

ALA is an intermediary in the heme pathway, forming the primary backbone of porphyrin synthesis, and it is another compound used for PDT. ${ }^{16}$ Exogenously delivered ALA appears to have selectivity for tumor tissues, possibly due to increased vascular permeability and decreased turnover by tumor cells. ${ }^{4}$ Since these initial developments, various other porphyrin, nonporphyrin and ALA based photosensitizers have become available for various specific diagnostic and therapeutic indications. ${ }^{16,17}$

PDT allows double targeting by mechanical and cellular mechanisms. Direct mechanical targeting is accomplished by placing the laser fiber directly into the tissue of interest, while cellular targeting is accomplished by the higher affinity of photosensitizers for accumulation in tumor cells, their organelles or their microvasculature. This multiple targeting confines treatment to a precise area.

An alternative to porphyrin based derivatives is bacteriochlorophyll. WST-09 is an agent from this novel family, in which the central magnesium ion is replaced by a palladium ion. WST-09 has had differential effects on tumor and nontumor tissues in preclinical studies and, in addition, the drug remains sequestered in the vascular compartment, resulting in significant improvements in drug toxicity, including rapid clearance and possibly better selectivity. ${ }^{18,19}$

Regarding toxicity, in a phase 1/2 trial of WST-09 in patients with locally recurrent prostate cancer after radiation therapy no skin phototoxicity was seen even when patients were challenged with skin light application. ${ }^{20}$ Regarding selectivity, one of the most unique properties of this compound is the vascular targeted application, whereby the most significant damage occurs to the microvasculature because the compound is restricted to the vascular space. This is evident in the current study, given the findings in the renal vasculature and particularly the glomeruli. Also, light penetrates more deeply into tissues due to the longer wavelength. On a practical level since illumination is performed within minutes of injection, no separate clinic visit or prolonged waiting time would be necessary, as is the case with most other compounds that are currently available.

This pilot study shows that VTP can be applied to the kidney and it results in localized tissue damage without affecting nontargeted renal tissue or overall renal function, which opens up the possibility of further exploring this novel application. Future efforts should use the water based version, WST-11, which would avoid the reactions due to the Cremophor solvent. ${ }^{8}$ The drug/light doses used in this pilot study were insufficient to cause consistent areas of necrosis. Thus, higher drug doses and light fluence should be tested to explore how the dynamic kinetics of drug and light interaction manifest in renal tissue. It is more than likely that multiple simultaneous interstitial laser fibers placed as an array would be necessary to treat larger areas, similar to brachytherapy for prostate cancer. Although the effect on tumor is expected to be more significant since prior studies have shown a preferential accumulation of photosensitizer in tumor microvasculature, this may be difficult to judge in preclinical trials due to the absence of a large animal model of renal cell carcinoma.

\section{CONCLUSIONS}

A significant, localized tissue effect was seen in all surviving animals, including limited necrosis at these relatively low drug/light combinations. VTP therapy of the kidney does not impair renal function. Aside from the acute anaphylactoid reaction seen in the initial animals, the treatment is well tolerated with no post-therapy side effects noted during the study period. A new formulation may make this treatment even safer. This study provides the initial proof of principle that VTP can function as a novel, minimally invasive nephron sparing modality for renal tumors. Further preclinical development is needed before any clinical trials.

\section{ACKNOWLEDGMENTS}

David J. Yang performed nuclear renography, Vickie Williams and Ginger Holloman assisted with the manuscript, and Negma-Lerads/Steba Biotech assisted with trial design. 


$\begin{aligned} & \text { Abbreviations and Acronyms } \\ & \text { ALA }=\text { aminolevulinic acid } \\ & \text { IM }=\text { intramuscular } \\ & \text { PDT }=\text { photodynamic therapy } \\ & \text { POD }=\text { postoperative day } \\ & \text { ROS }=\text { reactive oxygen species } \\ & \text { VTP }=\text { vascular targeted photodynamic therapy }\end{aligned}$

\section{REFERENCES}

1. Dougherty TJ: An update on photodynamic therapy applications. J Clin Laser Med Surg 2002; 20: 3.

2. Kereiakes DJ, Szyniszewski AM, Wahr D, Herrmann HC, Simon DI, Rogers C et al: Phase 1 drug and light doseescalation trial of motexafin lutetium and far red light activation (phototherapy) in subjects with coronary artery disease undergoing percutaneous coronary intervention and stent deployment. Circulation. 2003; 108: 1310.

3. Shackley DC, Briggs C, Whitehurst C, Betts CD, O'Flynn KJ, Clarke NW et al: Photodynamic therapy for superficial bladder cancer. Expert Rev Anticancer Ther 2001; 1: 523.

4. Frimberger D, Zaak D and Hofstetter A: Endoscopic fluorescence diagnosis and laser treatment of transitional cell carcinoma of the bladder. Semin Urol Oncol 2000; 18: 264.

5. Weersink RA, Bogaards A, Gertner M, Davidson SRH, Zhang $\mathrm{K}$, Netchev $\mathrm{G}$ et al: Techniques for delivery and monitoring of TOOKAD (WST09)-mediated photodynamic therapy of the prostate: clinical experience and practicalities. J Photochem Photobiol 2005; 79: 211.

6. Kramer B: Vascular effects of photodynamic therapy. Anticancer Res 2001; 21: 4271.

7. Mazor O, Brandis A, Plaks V, Neumark E, Rosenbach-Belkin V, Salomon Y et al: WST11, a novel water-soluble bacteriochlorophyll derivative; cellular uptake, pharmacokinetics, biodistribution and vascular-targeted photodynamic activity using melanoma tumors as a model. Photochem Photobiol 2005; 81: 342 .

8. Brandis A, Mazor O, Neumark E, Rosenbach-Belkin V, Salomon Y and Scherz A: Novel water-soluble bacteriochlorophyll derivatives for vascular-targeted photodynamic therapy: synthesis, solubility, phototoxicity and the effect of serum proteins. Photochem Photobiol 2005; 81: 983.

9. Svaasand LO: Photodynamic and photohyperthermic response of malignant tumors. Med Phys 1985; 12: 455.

10. Abels C: Targeting of the vascular system of solid tumours by photodynamic therapy (PDT). Photochem Photobiol Sci 2004; 3: 765.

11. Halliwell B and Gutteridge JMC: Free Radicals in Biology and Medicine. New York: Oxford University Press 1999.

12. Nieminen AL: Apoptosis and necrosis in health and disease: role of mitochondria. Int Rev Cytol Surv Cell Biol 2003; 224: 29 .

13. Agostinis P, Buytaert E, Breyssens H and Hendrickx N: Regulatory pathways in photodynamic therapy induced apoptosis. Photochem Photobiol Sci 2004; 3: 721.

14. Moan J and Peng Q: An outline of the hundred-year history of PDT. Anticancer Res 2003; 23: 3591.

15. Dougherty TJ, Gomer CJ, Henderson BW, Jori G, Kessel D, Korbelik M et al: Photodynamic therapy. J Natl Cancer Inst 1998; 90: 889.

16. Fukuda H, Casas A and Batlle A: Aminolevulinic acid: from its unique biological function to its star role in photodynamic therapy. Int J Biochem Cell Biol 2005; 37: 272.

17. Ackroyd R, Kelty C, Brown N and Reed M: The history of photodetection and photodynamic therapy. Photochem Photobiol 2001; 74: 656.

18. Vardi IY, Koudinova NV, Leibovitch I, Scherz A and Salomon Y: Photodynamic therapy of renal cell carcinoma (RCC) xenografts in mice, using a second generation photosensitizer-Tookad (WST09). J Urol 2004; 171: 261.

19. Huang Z, Chen Q, Luck D, Beckers J, Wilson BC, Trncic N et al: Studies of a vascular-acting photosensitizer, $\mathrm{Pd}$ bacteriopheophorbide (Tookad), in normal canine prostate and spontaneous canine prostate cancer. Lasers Surg Med 2005; 36: 390.

20. Weersink RA, Forbes J, Bisland S, Trachtenberg J, Elhilali M, Brún PH et al: Assessment of cutaneous photosensitivity of TOOKAD (WST09) in preclinical animal models and in patients. Photochem Photobiol 2005; 81: 106.

21. Spiegel MR: Mathematical Handbook of Formulas and Tables. Schaum's Outline Series in Mathematics. New York: McGraw-Hill Book Co 1968. 